Disponível em

http://www.anpad.org.br/rac

RAC, Curitiba, v. 15 , n. 6 , art. 7 ,

pp. 1100-1119, Nov./Dez. 2011

$((c)$ EY-NG

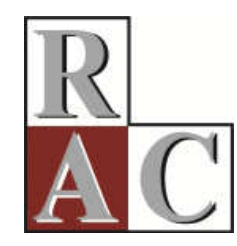

\title{
Gestão do Transporte Orientada para os Clientes: Nível de Serviço Desejado e Percebido
}

\section{Transportation Management Oriented for Customers: Service Level Desired and Perceived}

\author{
Ricardo Silveira Martins * \\ E-mail: martins@cepead.face.ufmg.br \\ Universidade Federal de Minas Gerais - CEPEAD/UFMG \\ Belo Horizonte, MG, Brasil. \\ Wescley Silva Xavier \\ E-mail: wescleysxavier@yahoo.com.br \\ Universidade Federal de Minas Gerais - CEPEAD/UFMG \\ Belo Horizonte, MG, Brasil. \\ Osmar Vieira de Souza Filho \\ E-mail: osmar.br@gmail.com \\ Universidade Federal de Minas Gerais - CEPEAD/UFMG \\ Belo Horizonte, MG, Brasil. \\ Guilherme Silveira Martins \\ E-mail: guilhermesmartins@gmail.com \\ Fundação Getúlio Vargas - EAESP/FGV \\ São Paulo, SP, Brasil.
}

* Endereço: Ricardo Silveira Martins

Av. Pres. Antônio Carlos, 6627, Belo Horizonte/MG, 31270-901.

Copyright (C) 2011 RAC. Todos os direitos, até mesmo de tradução, são reservados. É permitido citar parte de artigos sem autorização prévia, desde que seja identificada a fonte. 


\title{
Resumo
}

A gestão do transporte é tradicionalmente abordada na literatura como processo e seu fluxo, contextualizada em sua performance operacional e custos. Neste artigo, o transporte é analisado enquanto gestão de serviços, procurando entender aspectos comportamentais da demanda dos embarcadores. Foi realizado um survey com 400 embarcadores industriais brasileiros de pequeno e médio portes. A diferenciação das características dos serviços, segundo os clientes, foi obtida por Análise Fatorial. Os resultados indicaram que os construtos dos serviços de transporte mais valorizados pelas empresas embarcadoras foram, na ordem, Segurança, Confiabilidade, Tempo, Preço, Atendimento às necessidades especiais dos clientes e Relação com o cliente, ou seja, os embarcadores manifestaram maior interesse na eficácia do serviço. Desta forma, os embarcadores esperam que o desempenho do transporte por si reforce o relacionamento com seus clientes. Adicionalmente, foram detectadas diferenças nas prioridades, conforme os agrupamentos orientados pelas características dos clientes. A importância atribuída aos construtos Segurança e Confiabilidade pode também ajudar a compreender a razão de muitas empresas manterem frota própria ou instituírem contratos para a movimentação de seus produtos.

Palavras-chave: gestão de operações; logística; serviços de transporte; análise fatorial.

\begin{abstract}
Transport management is traditionally addressed in the literature as a process with its flow contextualized in its operating performance and costs. In this paper, transport is considered as service management, seeking to understand the behavioral aspects of the demand of shippers. We conducted a survey of 400 small and mediumsized Brazilian industrial shippers. The differentiation of services, according to the customers, was obtained by Factor Analysis. The results indicated that the constructs of transport services most valued by shippers were, in order, Security, Reliability, Time, Price, Service for the special needs of customers and Customer Relations, i.e., the shippers showed greater interest in the effectiveness of service. Thus, shippers expect the performance of transport in itself to strengthen the relationship with their customers. Additionally, we detected differences in priorities, according to the groups targeted by the characteristics of customers. The importance attached to the constructs of Security and Reliability can also help understand why many companies maintain their own fleet or establish contracts for the transport of their products.
\end{abstract}

Key words: operations management; logistics; transport service; factor analysis. 


\section{Introdução}

A gestão do transporte nas organizações implica a tomada de decisões sobre como movimentar materiais e produtos acabados entre diferentes pontos de uma determinada rede de negócios. Na abordagem tradicional, a gestão do transporte é discutida principalmente em questões operacionais de seus fluxos (Mason, Ribera, Farris, \& Kirk, 2003; Neuschel \& Russell, 1998; Ng, Ferrin, \& Pearson, 1997), medido em sua performance operacional e custos (McCann, 2001; Meixell \& Norbis, 2008).

Neste artigo, o transporte é analisado enquanto processos de gestão de serviços, procurando entender como os embarcadores de pequeno e médio porte formam suas expectativas quanto aos serviços, como estas diferem de acordo suas necessidades sob o ponto de vista dos seus clientes a serem atendidos e como estes serviços são avaliados. $\mathrm{O}$ ambiente estudado é o das pequenas e médias empresas industriais brasileiras.

Como componente da logística, as expectativas, quanto ao desempenho do serviço de transportes, se tornaram mais complexas. A busca da eficiência dos processos logísticos fez com que outros atributos do serviço fossem incorporados ao bojo da tomada de decisão acerca dos transportes, como o cumprimento dos prazos, a transparência de custos e o desenvolvimento de serviços apropriados e integrados com fornecedores e clientes.

A importância do transporte para as empresas pode ser entendida em sua capacidade de geração de valor de lugar, tanto em nível de serviços aos clientes quanto em sua contribuição na formação de custos. Assim, uma parcela importante da competitividade empresarial reside na correta elaboração e implementação de estratégias de transporte, com maior ou menor impacto, dependendo do tipo de negócio. Adicionalmente, tal estratégia de Operações deve estar alinhada à estratégia corporativa, conforme destacou o artigo seminal de Skinner (1969). Isto quer dizer decidir, entre as diversas alternativas, sobre os níveis de serviço (frequência e horários), propriedade da frota (própria ou transporte de terceiros) e a forma de consolidar as cargas (lotes e localização das operações).

Tendo como pano de fundo o impacto dos serviços de transportes no nível competitivo dos negócios, este estudo buscou analisar os fatores relevantes na tomada de decisão pela contratação e a satisfação dos embarcadores de pequeno porte em relação aos serviços de transporte. Embora o tema que envolve a atividade de transporte e sua importância na logística das empresas esteja desenvolvido nos aspectos da terceirização da atividade e contratação de operadores, ainda são registradas lacunas importantes quanto às suas especificidades para pequenos e médios negócios (Holter, Grant, Ritchie, \& Shaw, 2008).

Desta forma, a elucidação destas questões apresenta relevância gerencial e acadêmica. Em nível gerencial, aprofundar conhecimentos a respeito dos aspectos dos serviços de transportes, permite aprimoramentos futuros no processo de gestão, mais do que simples atos isolados de compras. No âmbito acadêmico, pelo fato da delimitação do estudo exploraram-se lacunas importantes no campo de estudo, abordando alinhamentos da gestão dos transportes como ativa gestão no processo dos negócios.

O artigo está organizado nas seguintes seções, a partir desta introdutória. A seção a seguir aborda aspectos da gestão da logística e dos transportes enquanto parte das estratégias de negócios, contextualizados no ambiente das pequenas e medias empresas. Em seguida, são apresentados detalhes da metodologia utilizada e, em seguida, são apresentados e discutidos os resultados. Finalmente, as conclusões, considerações e reconhecimento de limitações são feitos. 


\section{Gestão da Logística e dos Transportes como Fator Competitivo nas Organizações}

Segundo Neuschel e Russell (1998), a demanda dos serviços de transporte vem passando por transformações oriundas do ambiente concorrencial. Para os prestadores de serviços, tais transformações são percebidas na forma de aumento da competição, da necessidade de integração com prestadores de serviços de outras modalidades, para a movimentação de uma carga, do incentivo de mercado a novos entrantes, reflexo das baixas barreiras a entrada e da desregulação dos mercados, que implicou a liberdade para fixação de preço e agregação de serviços, e da pressão severa por preço, custo e melhoria do serviço por parte dos embarcadores.

Para os embarcadores, os serviços de transportes são, em geral, o pilar do composto da distribuição, pois impactam diretamente a satisfação do cliente. Numa situação de determinada organização produtos competitivos, a logística (entrega) pode impactar negativamente a avaliação global (produtos e serviços agregados) feita pelo cliente, por exemplo, podendo implicar perda de fidelidade ou não repetição da compra, conforme o desempenho das entregas em custo, nível de avarias, prazos e consistência geral dos serviços (Ballou, 2006).

As decisões do transporte mantêm interfaces diretas e significativas com as áreas financeira, de produção e de marketing (satisfação do cliente) das empresas. Na ótica financeira, segundo Mason, Ribera, Farris e Kirk (2003), estudos empíricos demonstraram que os custos com o transporte atingem de $2 \%$ a $4 \%$ do faturamento e de $30 \%$ a $60 \%$ dos custos logísticos totais das empresas. Conforme destacam Holter, Grant, Ritchie e Shaw (2008), além dos custos diretos, as decisões do transporte também implicam outros, tais como a formação de estoque, o nível de serviço e o planejamento da produção.

Decisões do transporte interagem com a produção, tanto para a disponibilidade de materiais quanto para produtos acabados. No que diz respeito aos materiais, a eficiência do transporte pode garantir que a produção planejada seja executada, contando com a disponibilidade de materiais para garantir a execução do processo. Além do mais, o cumprimento dos prazos acordados com os clientes dependerá também dos serviços de transporte contratados e executados (Holter et al., 2008).

Holter et al. (2008) chamam a atenção para a diferença que existe entre compra de serviços de transporte e gestão do transporte. O processo de aquisição dos serviços de transporte está envolvido no tradicional trade off entre custo e qualidade, que é resultante do processo de compra em si. Porém, a gestão do transporte implica o monitoramento do serviço desejado e adquirido, o que transcende as fronteiras da empresa, implicando fortemente a gestão de relacionamento com o operador.

Bowersox, Closs e Cooper (2002) sugerem que a gestão dos transportes deva ser executada pela avaliação dos serviços de transporte, baseados em parâmetros que permitam demonstrar o desempenho, por exemplo, em:

- Velocidade: Tempo gasto em trânsito;

- Disponibilidade: Capacidade de atender a qualquer origem e destino;

- Confiabilidade: Potencial de variação no tempo total de prestação do serviço;

Capacidade: Condição de manipular qualquer carga e em qualquer quantidade;

- Frequência: Capacidade de atender a qualquer momento.

Para harmonizar os serviços de transporte com as estratégias logísticas e de marketing, segundo Holter et al. (2008), os demandantes do transporte, os chamados embarcadores, baseiam a gestão dos processos do transporte nos aspectos custos de transporte, tempo em trânsito, rastreabilidade da carga, gestão das entregas e o custo total interno de gerenciamento da atividade. 
A gestão dos transportes no ambiente de negócios de menor porte tem algumas peculiaridades. Inicialmente, cabe destacar que a gestão de pequenas empresas tem forte componente familiar e de centralização na gestão. $\mathrm{O}$ dono da empresa envolve-se diretamente na gestão das atividades funcionais, entre elas o transporte. Para Gasse (1982), esse contato estreito com todas as atividades e o fato da visão da empresa estar fortemente concentrada no dono produz alguns efeitos na gestão, principalmente porque empreendedores requerem independência e autonomia.

Gasse (1982) ressalta que os resultados desse processo são a dificuldade de delegar, consultar e compartilhar atividades com terceiros, a lente voltada mais para a eficácia do que para a eficiência e a predileção excessiva do aproveitamento de oportunidades em detrimento de planejamento. Ou seja, não é de se esperar uma gestão especializada do transporte.

Para empresas de pequeno e médio porte, o transporte geralmente implica os maiores custos da logística, segundo Ng, Ferrin e Pearson (1997). Além do mais, é um processo repetido muitas vezes no cotidiano das empresas, tanto na compra de materiais quanto na distribuição de seus produtos.

Ainda conforme Holter et al. (2008), o transporte para negócios de pequeno porte também tem suas especificidades. Inicialmente, os embarcadores oferecem pequenos volumes aos operadores, o que implica, além de questão direta do poder de barganha nas negociações em preço e nível de serviço, maiores custos operacionais unitários ao operador, conforme McCann (2001). Ademais, o fator escala cria também dificuldades para a construção de um relacionamento mais harmonioso e equilibrado com os operadores de transporte, que permita às empresas internalizar as competências externas e transformá-las em força competitiva dos seus negócios (Grant, 2005).

Por outro lado, conforme Pappu, Mundy e Paswan (2001), as condições financeiras dos negócios de menor porte dificultam investimentos em ferramentas tecnológicas que viabilizem a gestão do transporte no conceito elucidado por Holter et al. (2008).

\section{Definições Metodológicas}

Este estudo é uma pesquisa empírica, de caráter exploratório, que procurou caracterizar as necessidades e expectativas dos embarcadores, quanto aos serviços das empresas de transporte rodoviário, no contexto dos pequenos e médios embarcadores industriais na realidade brasileira.

Essa pesquisa foi conduzida segundo critérios propostos por Collis e Hussey (2009): quanto aos seus objetivos constitui um estudo exploratório; quanto ao processo, este estudo é qualitativoquantitativo e; quanto à lógica, se classifica como pesquisa indutiva. $\mathrm{O}$ tipo de metodologia de procedimento é o survey, com amostragem não-probabilística por acessibilidade.

Malhotra (2001) destaca que a pesquisa exploratória é adequada em áreas de poucos conhecimentos acumulados, quando a compreensão dos fenômenos ainda não é suficiente ou até mesmo inexistente. Nesses casos, o caráter empírico da pesquisa apoia-se em levantamento de dados em campo e pela utilização de fontes de pesquisa complementares.

De acordo com Creswell (2003), estudos quali-quantitativos são caracterizados por reunir as técnicas e métodos de campo como observações e entrevistas a estudos tradicionais com dados quantitativos. Isso, de acordo com o autor, possibilita realizar uma triangulação entre as fontes de dados (quantitativos e qualitativos) e os resultados. Estudos de natureza quali-quantitativos envolvem análises empíricas e teóricas, de um caso particular, para a formulação de suposições que podem ser generalizadas.

A pesquisa teve caráter qualitativo, ao procurar compreender como se dá o gerenciamento da logística nas organizações estudadas. Nesse sentido, a pesquisa qualitativa tem por objetivo compreender um fenômeno por meio de uma análise holística, com a coleta de diferentes tipos de informações no contexto em que tal fenômeno ocorre (Yin, 1994). 
Quanto à lógica, utiliza a indução, que é a forma de abordagem do tema, em seu raciocínio e/ou argumentação, tendo como objetivo não só a produção de pensamentos, mas de orientar a reflexão.

\section{Amostra}

O processo de amostragem utilizado foi do tipo probabilístico, por tipicidade. A opção por essa forma de amostragem dá-se pela dificuldade de acesso às empresas. Collis e Hussey (2009) confirmam que, algumas vezes, é difícil obter uma amostra, particularmente quando se tratam de questões sensíveis ou que podem ser sigilosas. Para esses autores os dilemas éticos são barreiras para a delineação de uma amostra isenta de viés.

A coleta de dados ocorreu nos meses de fevereiro a abril de 2009, a partir da aplicação de questionários, in loco, em 400 empresas. Cabe ressaltar que, para evitar possíveis vieses de não resposta, optou-se aqui por realizar um filtro de dados ausentes, no qual a amostra foi reduzida para 384 empresas.

As empresas pesquisadas são de pequeno porte e foram caracterizadas pelo setor de atuação dentro da Indústria de transformação, conforme Classificação Nacional de Atividades Econômicas (CNAE, versão 2.0), do Conselho Nacional de Classificação, utilizada pela Fundação Instituto Brasileiro de Geografia e Estatística (IBGE).

A unidade de análise de pesquisa refere-se às empresas da indústria de transformação de Minas Gerais de até 100 funcionários, tendo por base o Cadastrado de Estabelecimentos Empregadores (CEE) do Ministério do Trabalho. As unidades de observação foram os empresários e gerentes envolvidos no processo de planejamento e gestão do transporte.

No contexto brasileiro, as pequenas e médias empresas (PMEs) possuem importância destacada, pois representam 99,2\% das empresas registradas, geram 57,2\% dos empregos formais e respondem por $20 \%$ do Produto Interno Bruto (PIB) (Serviço de Apoio às Micro e Pequenas Empresas [SEBRAE], 2009).

\section{Estratégias de pesquisa, coleta de dados e natureza das informações}

A coleta de dados ocorreu por meio de entrevista com questionário. A estratégia de pesquisa adotada foi a visita in loco, através de Survey. Cervo, Bervian e Silva (2007) comentam que esta técnica é utilizada para obter informações que não podem ser encontradas em registros ou fontes documentais, mas que podem ser fornecidas por alguém. A coleta de dados ocorreu nos meses de fevereiro a abril de 2009.

Neste questionário, inseriram-se questões que tratavam dos construtos e atributos do transporte, bem como do nível de satisfação com o serviço. Para a elaboração do instrumento de pesquisa, foram buscadas as orientações teóricas em torno das dimensões relevantes à avaliação do transporte em Novaes (2001), Schluter e Senna (1999) e Valente, Passaglia e Novaes (2008). Além de revisão teórica, o instrumento de pesquisa também utilizou a experiência de estudos anteriores que tiveram propósitos semelhantes, tais como Agência Nacional de Transporte Terrestre (ANTT, 2005) e Banco Internacional de Reconstrução e Desenvolvimento; Agência Nacional de Transporte Terrestre (BIRD/ANTT, 2006).

A avaliação do transporte considerou aspectos do processo de contratação dos serviços de transporte, dos construtos que orientam a tomada de decisão e da avaliação dos serviços disponíveis e efetivamente utilizados. Os fatores que influenciam tanto a escolha quanto a avaliação do serviço prestado foram elaborados conforme os construtos que foram tratados na seção anterior, quando foram elencadas as principais referências na gestão da logística das empresas, em torno dos construtos apresentados por Bowersox, Closs e Cooper (2002), das preocupações com a qualidade dos serviços e seus impactos nas avaliações dos embarcadores (Ballou, 2006), a dimensão financeira avaliada por Mason et al. (2003) e instrumentos empíricos desenvolvidos para a análise da qualidade e da 
satisfação com os serviços de transporte no Brasil, como aquele desenvolvido por ANTT (2005). Desta forma, a análise da avaliação dos serviços de transporte e da satisfação quanto a eles por parte do embarcador orbitou em torno dos seguintes construtos:

- Tempo, entendido como o tempo gasto entre contratação do serviço e a efetiva finalização do serviço contratado (coleta/entrega da carga);

- Segurança no transporte e da carga;

· Confiabilidade no serviço: variações do serviço efetivo em relação ao contratado;

- Preço;

- Relação com o cliente (atendimento e troca de informações com o cliente);

- Atenção às necessidades especiais do cliente (procedimentos de carga/descarga ou questões relativas ao atendimento do fornecedor/cliente).

Quanto aos aspectos relativos à escolha da transportadora para todas as empresas da amostra, verificaram-se as notas médias, em escala de 1 a 5, atribuídas aos fatores Tempo, Segurança, Confiabilidade, Preço, Relação com cliente e Atendimento às necessidades especiais dos clientes. Esta análise foi desenvolvida para o conjunto das empresas e para os agrupamentos de empresas, com base nos comportamentos similares e na escala demandada do transporte. Estas duas últimas situações serão detalhadas mais à frente.

A escala de 1 a 5 também foi utilizada para que os embarcadores avaliassem os serviços atualmente disponibilizados pelo mercado. Nestes casos, foi utilizada uma escala linear horizontal não estruturada. A vantagem da utilização da escala linear não estruturada é a ausência de um valor numérico ao longo da escala, objetivando maior isenção às respostas (Stone \& Sidel, 1993).

Para avaliar eventuais diferenças no comportamento das empresas, guardando particularidades com seus produtos, clientes e segmentos de mercado, foram definidos padrões de serviços, priorizando construtos e atributos do transporte, pelo uso da Análise fatorial Exploratória. A análise fatorial pode ser definida como técnica estatística multivariada utilizada para estudar o inter-relacionamento de variáveis observadas, a fim de definir um conjunto de dimensões latentes comuns, chamadas de fatores (Hair, Anderson, Tatham, \& Black, 2005).

As mesmas análises foram desenvolvidas para grupos formados a partir da orientação da escala dos serviços necessários: tipicamente, carga completa ou carga fracionada. A investigação objetivou buscar evidências de comportamentos diferenciados na demanda pelo transporte, conforme escala de operações. As empresas foram organizadas em dois grupos, conforme setores de atividade econômica e clientes típicos (Tabela 1).

Tabela 1

\section{Grupos de Embarcadores, Conforme Escala do Transporte}

\begin{tabular}{cccc}
\hline Grupos & Setores de atividade econômica & Cliente típico & $\begin{array}{c}\text { Número de } \\
\text { empresas }\end{array}$ \\
\hline $\mathbf{1}$ & $\begin{array}{c}\text { Metalurgia, Bens Industrializados } \\
\text { Intermediários e Tecnologia }\end{array}$ & $\begin{array}{c}\text { Atacadistas, distribuidores e indústrias } \\
\text { (pedidos de maior volume) }\end{array}$ & 112 \\
$\mathbf{2}$ & Alimentos e Vestuários e Calçados & $\begin{array}{c}\text { Varejo e o consumidor final (pedidos de } \\
\text { menor volume) }\end{array}$ & 183 \\
\hline
\end{tabular}

Nota. Fonte: Elaboração dos autores. 
A fim de melhor elucidar os procedimentos de análise adotados neste trabalho, propomos uma estrutura analítica que visa ressaltar a operacionalização dos atributos e variáveis, bem como os procedimentos de análise estatística aqui utilizados (Figura 1).

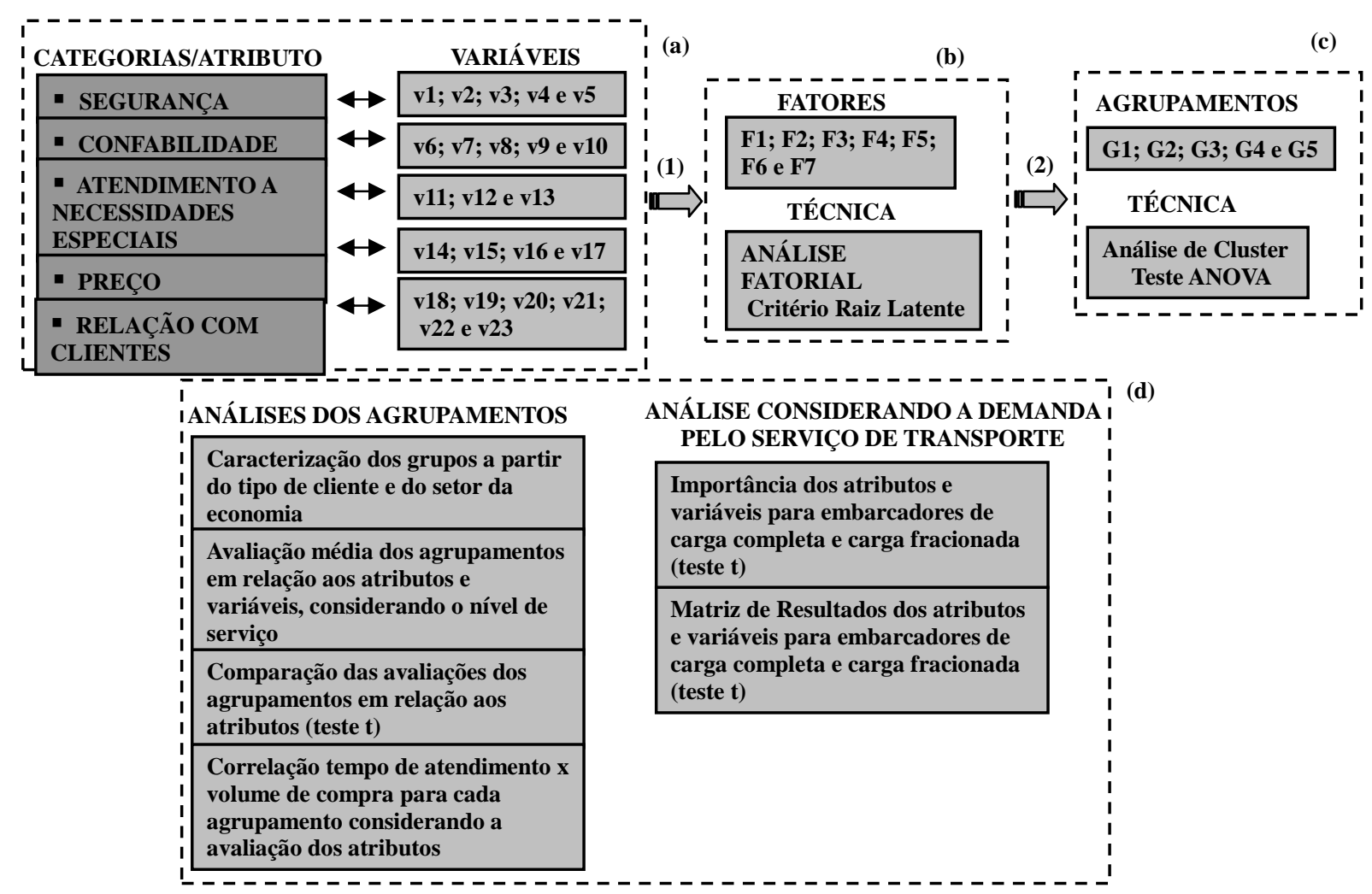

Figura 1. Estrutura Analítica da Operacionalização das Variáveis e Procedimentos de Análise.

As categorias/atributos oriundas da revisão bibliográfica são formadas por variáveis diversas (ver Tabela 3). Em síntese, é a combinação destas variáveis que caracteriza uma categoria ou um atributo (a), caracterizando uma inter-relação. Dada a possibilidade de identificar a aproximação das variáveis, mesmo aquelas que a priori se originam de categorias distintas. De forma a identificar esta aproximação entre as variáveis, a primeira operacionalização realizada é a Análise Fatorial Exploratória (1).

Com o uso da Análise Fatorial, considerando o critério da raiz latente (b), foram obtidos sete fatores, cada um representando a aproximação estatística entre as variáveis. Em um segundo momento, estes sete fatores foram condensados em cinco agrupamentos (2). Estes agrupamentos foram estatisticamente formados com o uso da Análise de Cluster e do teste ANOVA (c), nos quais são parâmetros comportamentos semelhantes entre as empresas, a partir do peso dos fatores que, por sua vez, foram gerados pela avaliação das variáveis. Cabe ressaltar mais uma vez que a principal característica do agrupamento é apresentar homogeneidade entre os que o compõem, e heterogeneidade entre os membros que compõem outro cluster, sendo esta última característica assegurada pelo teste ANOVA.

Após a formação dos agrupamentos, buscou-se caracterizar e descrever as diferenças existentes entre empresas de cada grupo. Entre os fatores relevantes na seleção e uso do serviço de transporte encontrados na literatura, destacam-se os tipos de clientes e o setor da economia, uma vez que não apenas o porte do cliente, mas também o maior ou de menos grau de especificidade - variando conforme o setor de atuação - podem representar diferenças na relevância de critérios de seleção e avaliação do serviço de transporte. 
Ademais, as análises contemplam as avaliações dos atributos e variáveis para os diferentes agrupamentos formados (diferenças estatísticas asseguradas com o uso do teste t), e a correlação entre tempo de atendimento e volume de compra, bem como esta relação para graus diversos de importância atribuídos a cada um dos atributos. Por fim, e também com a utilização do teste de diferença de médias $t$, buscou-se evidenciar possíveis diferenças na avaliação do nível de serviço das transportadoras, na comparação entre embarcadores de carga completa e embarcadores de carga fracionada, uma vez que estes últimos tendem a ter reduzido o poder de barganha; portanto aparentemente são suscetíveis a níveis de serviço mais precários.

\section{Resultados e Discussão}

Em seguida, são apresentados os resultados, iniciando-se pelos agrupamentos formados com base nos comportamentos dos embarcadores. Posteriormente, estes comportamentos são devidamente contextualizados e discutidos enquanto processo logístico estratégico.

\section{Agrupamentos de embarcadores, conforme aspectos comportamentais}

A análise fatorial das variáveis utilizadas foi validada pelo teste de esfericidade de Barlett e pelo índice de Kaiser-Meyer-Olkin (KMO). O primeiro testa a probabilidade da matriz de correlação ser uma matriz identidade, o que indicaria inadequação da análise fatorial. Já o índice de KMO mede a adequação da amostra quanto à correlação das variáveis. Hair, Anderson, Tatham e Black (2005) alertam para a necessidade de que o índice de KMO seja superior a 0,5. Para esse estudo, verificou-se um índice de KMO de 0,797, o que indica a adequação da amostra. Em relação ao teste de esfericidade de Barlett, verificou-se o nível de significância de 0,000, o que permite rejeitar a hipótese de matriz identidade para as correlações entre variáveis.

No que diz respeito à definição do número de fatores a serem extraídos, a partir das variáveis, foi utilizado o critério da raiz latente, de forma que cada fator explicasse, pelo menos, sua própria variância. Dessa forma, chegou-se ao número de sete fatores. Cada um dos sete fatores é composto por um conjunto de variáveis, sendo cada uma exclusiva de um único fator.

A importância de agrupar atributos se deve à possibilidade de criar dimensões - que aqui são denominados fatores - que podem demonstrar que seja importante na prestação do serviço. Como exemplo, vale ressaltar a possibilidade de ter uma dimensão ou fator Preço. Este fator poderia ser composto de diversas variáveis, e não apenas o preço em si, como política de descontos, prazos, formas de pagamento etc. A técnica de análise fatorial permite, então, agrupar estes elementos que irão constituir a dimensão ou fator preço. O conjunto de dimensões explica todo o fenômeno; neste caso, o que os entrevistados acreditam ser importante no serviço de transporte.

De acordo com a Tabela 2, os fatores ou dimensões do modelo encontrados explicam juntos $62,415 \%$ variância total do modelo. Isso significa que os fatores aqui formados conseguem explicar mais da metade do que seria considerado pelas empresas como importante no serviço de transporte, sendo este resultado estatisticamente relevante. 
Tabela 2

Total da Variância Explicada em Relação à Importância de Atributos do Serviço de Transporte

\begin{tabular}{|c|c|c|c|c|c|c|c|c|c|}
\hline \multirow[t]{2}{*}{ Fatores } & \multicolumn{3}{|c|}{ Autovalores } & \multicolumn{3}{|c|}{ Somatório das cargas extraídas } & \multicolumn{3}{|c|}{ Somatório após a rotação } \\
\hline & Total & $\begin{array}{c}\% \text { de } \\
\text { variância }\end{array}$ & $\underset{\%}{\text { Acumulado }}$ & Total & $\begin{array}{c}\% \text { de } \\
\text { variância }\end{array}$ & $\underset{\%}{\text { Acumulado }}$ & Total & $\begin{array}{c}\text { \% de } \\
\text { variância }\end{array}$ & $\underset{\%}{\text { Acumulado }}$ \\
\hline 1 & 5,925 & 25,762 & 25,762 & 5,925 & 25,762 & 25,762 & 2,886 & 12,546 & 12,546 \\
\hline 2 & 1,943 & 8,448 & 34,210 & 1,943 & 8,448 & 34,210 & 2,421 & 10,528 & 23,074 \\
\hline 3 & 1,609 & 6,998 & 41,208 & 1,609 & 6,998 & 41,208 & 2,222 & 9,662 & 32,736 \\
\hline 4 & 1,402 & 6,097 & 47,304 & 1,402 & 6,097 & 47,304 & 1,842 & 8,007 & 40,742 \\
\hline 5 & 1,283 & 5,580 & 52,885 & 1,283 & 5,580 & 52,885 & 1,698 & 7,383 & 48,125 \\
\hline 6 & 1,135 & 4,934 & 57,818 & 1,135 & 4,934 & 57,818 & 1,649 & 7,168 & 55,294 \\
\hline 7 & 1,057 & 4,597 & 62,415 & 1,057 & 4,597 & 62,415 & 1,638 & 7,121 & 62,415 \\
\hline
\end{tabular}

Nota. Fonte: Resultados da Pesquisa.

As variáveis agrupadas formam os sete fatores, como pode ser observado na Tabela 3. O primeiro fator trata do cuidado com a carga, podendo ser motivado pelo valor da carga e/ou pela necessidade de cumprimento estrito de contratos. No segundo fator estão presentes duas características inter-relacionadas, no caso, aspectos da comunicação entre a empresa demandante do serviço e a transportadora, e a responsividade da transportadora em solucionar os problemas dos clientes, particularmente no que diz respeito à cooperação técnica. $\mathrm{O}$ terceiro fator diz respeito ao pagamento pelo serviço de transporte, seja o valor, as formas ou a necessidade de empresas concorrerem entre si com fins de redução de preço. Representa preocupações essencialmente como os custos do serviço para o embarcador. $\mathrm{O}$ quarto fator remete à necessidade da carga ser entregue sem avarias e no momento certo. São variáveis típicas de relacionamentos que baseiam-se na responsividade, em que erros podem representar a paralisação da produção ou perda de clientes. Quanto ao quinto fator, a preocupação manifestada como prioritária é quanto à regularidade e flexibilidade operacional. Já o sexto fator representa a possibilidade de controle e segurança da carga e do que fora acordado. Por fim, o sétimo fator que se caracteriza pela prioridade de transparência e da segurança na prestação do serviço.

Após a formação dos fatores a partir da análise fatorial, procedeu-se à formação de clusters, que representa então a possibilidade de aproximação entre fatores, com base na escolha de uma medida de semelhança ou distância entre as observações e de um procedimento de agrupamentos fundamentados em suas medidas. O objetivo específico desta análise constitui-se na formação de clusters com características de homogeneidade dentro deles e heterogeneidade entre eles, ou pequenas variações dentro do cluster em relação às variações entre os clusters (Dillon \& Goldstein, 1984).

Tabela 3

Distribuição das Variáveis nos Fatores

\begin{tabular}{|c|c|c|c|c|c|c|c|}
\hline \multirow{2}{*}{ Variáveis } & \multicolumn{7}{|c|}{ Fatores } \\
\hline & 1 & 2 & 3 & 4 & 5 & 6 & 7 \\
\hline (V1) Importância da prevenção a roubo & ,744 & & & & & & \\
\hline (V2) Importância da prevenção a avarias & ,819 & & & & & & \\
\hline (V3) Importância dos caminhões & 604 & & & & & & \\
\hline (V4) Importância da prevenção a acidentes & 660 & & & & & & \\
\hline
\end{tabular}




\section{Tabela 3 (continuação)}

\begin{tabular}{|c|c|c|c|c|c|c|c|}
\hline \multirow{2}{*}{ Variáveis } & \multicolumn{7}{|c|}{ Fatores } \\
\hline & 1 & 2 & 3 & 4 & 5 & 6 & 7 \\
\hline (V5) Importância do ressarcimento a prejuízos & ,682 & & & & & & \\
\hline (V6) Importância do cumprimento do contrato & & & & & & ,632 & \\
\hline (V7) Importância da regularidade no serviço & & & & &, 577 & & \\
\hline $\begin{array}{l}\text { (V8) Importância da capacidade de distribuir } \\
\text { caminhões pelo país }\end{array}$ & & & & &, 742 & & \\
\hline (V9) Importância da pontualidade na entrega & & & & ,858 & & & \\
\hline (V10) Importância da integridade da carga & & & & ,876 & & & \\
\hline (V11) Importância do Pessoal da Transportadora & & & & & & & ,799 \\
\hline (V12) Importância das características operacionais & & & & & ,342 & & \\
\hline $\begin{array}{l}\text { (V13) Importância da disponibilidade de } \\
\text { informações }\end{array}$ & & & & & & & ,617 \\
\hline (V14) Importância do valor do frete & & &, 555 & & & & \\
\hline (V15) Importância das políticas de descontos & & & ,766 & & & & \\
\hline (V16) Importância dos prazos de pagamento & & & ,774 & & & & \\
\hline $\begin{array}{l}\text { (V17) Importância da competitividade entre } \\
\text { concorrentes }\end{array}$ & & &, 572 & & & & \\
\hline $\begin{array}{l}\text { (V18) Importância da facilidade de comunicação } \\
\text { com a transportadora }\end{array}$ & & ,691 & & & & & \\
\hline (V19) Importância da agilidade no serviço & & ,729 & & & & & \\
\hline $\begin{array}{l}\text { (V20) Importância da autonomia do responsável no } \\
\text { atendimento }\end{array}$ & & ,622 & & & & & \\
\hline $\begin{array}{l}\text { (V21) Importância da eficiência na solução de } \\
\text { problemas }\end{array}$ & & 683 & & & & & \\
\hline $\begin{array}{l}\text { (V22) Importância de sistemas de rastreamento via } \\
\text { internet }\end{array}$ & & & & & & ,637 & \\
\hline $\begin{array}{l}\text { (V23) Importância da cooperação técnica com } \\
\text { clientes }\end{array}$ & & ,408 & & & & & \\
\hline
\end{tabular}

Nota. Fonte: Resultados da Pesquisa.

Cabe ressaltar que as médias encontradas nos construtos para cada agrupamento foram submetidas ao teste ANOVA, que avalia a significância estatística da diferença entre duas médias. Este teste foi utilizado para averiguar a certeza dos comportamentos diferenciados entre os embarcadores. Dessa forma, os testes foram realizados em pares, sendo significantes, isto é, com médias dos atributos diferentes, segundo os seguintes clusters apresentados na Tabela 4. 
Tabela 4

Resultados do Teste ANOVA

\begin{tabular}{cccccccc}
\hline \multirow{2}{*}{ Factor Score } & \multicolumn{9}{c}{ Cluster } & \multicolumn{2}{c}{ Error } & \multirow{2}{*}{ F } & Sig. \\
\cline { 2 - 5 } & Mean Square & df & Mean Square & df & & \\
\hline 1 & 59,543 & 4 &, 382 & 379 & 155,821 &, 000 \\
2 & 41,672 & 4 &, 571 & 379 & 73,015 &, 000 \\
3 & 2,852 & 4 &, 980 & 379 & 2,908 &, 022 \\
4 & 52,744 & 4 &, 454 & 379 & 116,205 &, 000 \\
5 & 2,770 & 4 &, 981 & 379 & 2,823 &, 025 \\
6 & 51,985 & 4 &, 462 & 379 & 112,546 &, 000 \\
7 & 3,094 & 4 &, 978 & 379 & 3,163 &, 014 \\
\hline
\end{tabular}

Nota. Fonte: Resultados da Pesquisa.

A Tabela 4 indica a relação entre os fatores gerados na análise fatorial e os clusters. Os valores nas intersecções na tabela representam os escores, de forma que possam ser atribuídas características aos agrupamentos. Vale ressaltar que um fator, em sua natureza, já é composto por variáveis que exprimem características diversas.

Dessa forma, ao serem alocados em um número de agrupamentos ainda menor (cinco, ao invés de sete), existe a possibilidade de um único fator estar presente em dois ou mais agrupamentos, bem como um único agrupamento ter características de dois ou mais fatores, como pode ser observado na Tabela 5, em termos de escores.

Tabela 5

Escores de Atração dos Clusters

\begin{tabular}{cccccc}
\hline \multirow{2}{*}{ Fatores } & \multicolumn{5}{c}{ Clusters } \\
\cline { 2 - 6 } & $\mathbf{1}$ & $\mathbf{2}$ & $\mathbf{3}$ & $\mathbf{4}$ & $\mathbf{5}$ \\
\hline $\mathbf{1}$ & 0,04927 & $-2,60505$ & 0,18090 & 0,23385 & 0,38112 \\
$\mathbf{2}$ & 0,10171 & $-0,22129$ & 0,09641 & 0,28091 & $-1,99906$ \\
$\mathbf{3}$ & 0,27830 & $-0,09229$ & 0,14112 & 0,04515 &,- 49748 \\
$\mathbf{4}$ & $-4,29715$ & $-0,09497$ & 0,14320 & 0,14001 & 0,20755 \\
$\mathbf{5}$ & $-0,86519$ & $-0,14295$ & $-0,02540$ & 0,02541 & 0,23545 \\
$\mathbf{6}$ & $-0,35918$ & 0,21292 & $-2,06737$ & 0,31094 & 0,06082 \\
$\mathbf{7}$ & $-0,29469$ & $-0,00521$ &,- 29370 & 0,10913 & $-0,35991$ \\
\hline
\end{tabular}

Nota. Fonte: Resultados da Pesquisa.

\section{Construtos dos serviços de transporte segundo os embarcadores}

Para efeito de análise, optou-se por definir previamente o número de clusters em cinco. A escolha de cinco clusters se justifica pela possibilidade de equilíbrio entre homogeneidade dentro do cluster e heterogeneidade entre os clusters. Caso a opção pelo número de clusters fosse superior a cinco, haveria a possibilidade de um menor número de empresas por cluster, sem atratividade entre 
variáveis, o que reduziria a heterogeneidade entre os clusters. Do contrário, ou seja, um baixo número de clusters, aconteceria a redução da homogeneidade dentro do cluster.

Como os fatores gerados na análise fatorial são constituintes dessas características, entre outras que serão utilizadas para análise descritiva, os escores dos fatores foram utilizados para a caracterização inicial dos agrupamentos (clusters). Desta forma, os clusters formados são aqueles apresentados na Tabela 6 .

Tabela 6

Clusters Formados com o Grupo de Empresas da Amostra

\begin{tabular}{ccc}
\hline Cluster & Clientes típicos & Setores \\
\hline $\mathbf{1}$ & Empresas industriais e de distribuição, em todo o & território \\
$\mathbf{2}$ & Varejo local & Metalurgia Tecnologia \\
$\mathbf{3}$ & Varejo geral, cliente final e empresas industriais & Vestuário e Calçados \\
$\mathbf{4}$ & Varejo de todo o território & $\begin{array}{c}\text { Alimentos, Móveis e Bens } \\
\text { Industrializados Intermediários }\end{array}$ \\
$\mathbf{5}$ & Atacadistas, grande varejo e indústrias nacionais & Alimentos e Vestuário e Calçados \\
\hline
\end{tabular}

Nota. Fonte: Resultados da Pesquisa.

Segundo Meixel e Norbis (2008), os critérios mais seguidos na contratação de serviços de transporte, em escala mundial, são os de Confiança no serviço, Tempo em trânsito (Tempo) e Custos logísticos, em primeiro plano, seguidos de Índice de avarias, Disponibilidade de veículo/ flexibilidade do serviço, Atendimento, Frete e Qualidade do serviço.

Com base na Tabela 6, no caso geral, é possível observar que os construtos de maior importância foram a Segurança e a Confiabilidade nos serviços prestados pelas transportadoras. Há que ressaltar que as menores médias foram encontradas nos critérios Relação com o cliente e Atendimento a necessidades especiais dos clientes (Tabela 7).

Tabela 7

Avaliação dos Serviços de Transporte, Segundo os Construtos e os Critérios dos Clusters

\begin{tabular}{lccccccc}
\hline \multirow{2}{*}{ Construtos } & Média & \multicolumn{5}{c}{ Clusters } \\
\cline { 3 - 8 } & Geral & $\mathbf{1}$ & $\mathbf{2}$ & $\mathbf{3}$ & $\mathbf{4}$ & $\mathbf{5}$ \\
\hline Tempo & 4,45 & 4,00 & 4,44 & 4,24 & 4,53 & 4,31 \\
Segurança (no transporte e na carga) & 4,60 & 3,64 & 3,75 & 4,48 & 4,75 & 4,75 \\
Confiabilidade & 4,59 & 4,27 & 4,03 & 4,48 & 4,68 & 4,61 \\
Preço & 4,34 & 4,18 & 4,03 & 4,43 & 4,36 & 4,28 \\
Relação com o cliente & 4,23 & 3,36 & 4,06 & 3,79 & 4,37 & 4,19 \\
Atendimento a necessidades & & & & & & \\
especiais dos clientes & 4,28 & 4,09 & 3,69 & 3,86 & 4,42 & 4,28 \\
\hline
\end{tabular}

Nota. Fonte: Resultados da Pesquisa.

Ou seja, a Segurança é a principal característica dos serviços de transporte. Tal nível de importância pode ser interpretado como a alta dependência dos embarcadores, quanto à necessidade da 
execução dos serviços de transporte, de forma que o cliente receba a mercadoria. Desse modo, esperam que o transporte não seja fonte de desgastes perante o cliente, sinalizando até mesmo um trade off com o preço, à medida que sinalizam ser este um atributo em $4^{\circ}$ nível de importância.

Cabe ressaltar que as médias encontradas nos construtos para cada agrupamento foram submetidas ao teste $t$, que avalia a significância estatística da diferença entre duas médias. Este teste foi utilizado para averiguar a certeza dos comportamentos diferenciados entre os embarcadores. Dessa forma, os testes foram realizados em pares, sendo significantes, isto é, com médias dos atributos diferentes segundo os seguintes clusters apresentados na Tabela 8.

Tabela 8

Diferença de Médias dos Construtos entre Clusters

\begin{tabular}{cl}
\hline Clusters comparados & \multicolumn{1}{c}{ Construtos com médias estatisticamente diferentes ${ }^{\mathbf{1}}$} \\
\hline 1 e 2 & Tempo, Segurança, Confiabilidade, Atendimento às necessidades especiais \\
1 e 3 & Confiabilidade, Atendimento às necessidades especiais \\
1 e 4 & Tempo, Atendimento às necessidades especiais \\
1 e 5 & Tempo, Segurança, Atendimento às necessidades especiais \\
2 e 3 & Segurança \\
2 e 4 & Segurança, Confiabilidade, Atendimento às necessidades especiais \\
2 e 5 & Segurança, Confiabilidade \\
3 e 4 & $\begin{array}{l}\text { Tempo, Segurança, Confiabilidade, Relação com cliente, Atendimento às } \\
\text { necessidades especiais }\end{array}$ \\
3 e 5 & Tempo, Segurança, Confiabilidade \\
4 e 5 & -
\end{tabular}

Nota. 1-Ao nível de 5\% de significância (teste t).

Fonte: Resultados da Pesquisa.

Por outro lado, pode-se dizer também que as características dos serviços são variáveis. À medida que o volume do pedido aumenta, os embarcadores passam a ser mais exigentes quanto ao desempenho dos construtos Confiabilidade, Preço e Atenção às necessidades especiais (Tabela 9). Tal constatação foi obtida das correlações positivas encontradas entre estes construtos.

Tabela 9

Correlação Volume do Pedido versus Prazo Médio de Atendimento e Avaliação Geral

\begin{tabular}{lccccc}
\hline \multirow{2}{*}{ Construtos } & \multicolumn{5}{c}{ Clusters } \\
\cline { 2 - 6 } & $\mathbf{1}$ & $\mathbf{2}$ & $\mathbf{3}$ & $\mathbf{4}$ & $\mathbf{5}$ \\
\hline Tempo & 0,443 & $-0,045$ & $-0,039$ & $-0,015$ & $-0,067$ \\
Segurança & $-0,170$ & $-0,030$ & 0,048 & 0,053 & $-0,147$ \\
Confiabilidade & $-0,205$ & 0,252 & 0,024 & 0,078 & 0,098 \\
Preço & 0,393 & 0,423 & $-0,137$ & 0,021 & $-0,007$ \\
Relacionamento com cliente & $-0,089$ & 0,092 & 0,080 & $-0,003$ & $-0,069$ \\
Atenção às necessidades especiais & $-0,046$ & $-0,051$ & 0,149 & 0,086 & 0,124 \\
\hline
\end{tabular}

Nota. Fonte: Resultados da Pesquisa. 
Ou seja, as empresas passam a dedicar atenção ao planejamento, desempenho, formatação do nível de serviço e negociação de preços à medida que os volumes a serem movimentados aumentam. Aí o transporte passa a ser mais importante do ponto de vista do embarcador.

Os embarcadores sinalizam que o transporte se torna estratégico apenas quando altos valores financeiros estão sendo movimentados em mercadorias, e não como parte do processo logístico por si. Por outro lado, tal situação remete a uma crença errônea de que logística seja estratégica apenas para grandes negócios, devendo apenas, nestes casos, ter caráter estratégico.

Além disso, foram encontradas diferenças, quando as análises recaem sobre os clusters. Para as empresas do cluster 1, o aumento do pedido eleva a importância dos construtos Tempo e Preço. Já para as empresas do cluster 2, a importância se eleva para os construtos Confiabilidade, Preço e Relacionamento com o cliente. Entre as empresa do cluster 3, só não há relação direta para os construtos Tempo e Preço. Da mesma forma, as empresas do cluster 4 só não aumentam o grau de exigência com o aumento dos pedidos nas variáveis Tempo e Relacionamento com os clientes. Por fim, as empresas do cluster 5 são mais exigentes para os construtos Confiabilidade e Atenção às necessidades especiais dos clientes (Tabela 9).

Além da importância dos atributos na escolha da transportadora, buscou-se também verificar a satisfação do serviço prestado. A Tabela 10 apresenta as avaliações gerais e dos clusters a cada um dos atributos avaliados numa escala de 1 a 5 .

Tabela 10

Satisfação com Transporte, segundo Avaliação dos Clusters de Embarcadores

\begin{tabular}{|c|c|c|c|c|c|c|c|}
\hline \multirow{2}{*}{ Construtos } & \multirow{2}{*}{ Atributos } & \multirow{2}{*}{$\begin{array}{l}\text { Média } \\
\text { Geral }\end{array}$} & \multicolumn{5}{|c|}{ Clusters } \\
\hline & & & 1 & 2 & 3 & 4 & 5 \\
\hline \multirow[t]{3}{*}{ Tempo } & $\begin{array}{l}\text { Tempo para cotar prazo, especificação } \\
\text { e preço }\end{array}$ & 3,96 & 3,03 & 3,79 & 3,95 & 4,03 & 3,76 \\
\hline & Tempo para iniciar atendimento & 4,20 & 3,37 & 4,00 & 4,13 & 4,35 & 4,00 \\
\hline & Tempo para entregar produto & 4,25 & 3,34 & 3,82 & 4,53 & 4,35 & 4,34 \\
\hline Segurança & Nível baixo de avarias nas cargas & 4,46 & 3,12 & 4,86 & 4,28 & 4,57 & 4,30 \\
\hline \multirow[t]{2}{*}{ Confiabilidade } & Cumprimento dos prazos & 4,13 & 2,98 & 3,83 & 4,28 & 4,25 & 4,08 \\
\hline & Percentual de entrega nos prazos & 4,50 & 3,36 & 4,36 & 4,63 & 4,64 & 4,42 \\
\hline Preço & Preço & 4,13 & 3,91 & 3,97 & 4,41 & 4,14 & 4,21 \\
\hline \multirow[t]{4}{*}{$\begin{array}{l}\text { Relacionamento } \\
\text { com o cliente }\end{array}$} & $\begin{array}{l}\text { Disposição para ouvir e agir no caso } \\
\text { de comunicação de falhas/problemas }\end{array}$ & 3,83 & 3,26 & 3,54 & 3,96 & 3,96 & 3,66 \\
\hline & Informações sobre o status da carga & 3,88 & 2,69 & 3,61 & 3,85 & 4,07 & 3,47 \\
\hline & Atendimento educado e cortês & 4,58 & 3,74 & 4,51 & 4,74 & 4,69 & 4,29 \\
\hline & Atendimento a qualquer estado do Brasil & 3,94 & 3,44 & 3,67 & 4,49 & 3,96 & 3,55 \\
\hline \multirow[t]{2}{*}{$\begin{array}{l}\text { Atenção às } \\
\text { necessidades } \\
\text { especiais }\end{array}$} & $\begin{array}{l}\text { Desenvolvimento dos serviços } \\
\text { Conforme especificação }\end{array}$ & 4,31 & 3,07 & 4,18 & 4,51 & 4,38 & 4,32 \\
\hline & Média geral & 4,18 & 3,28 & 4,01 & 4,31 & 4,28 & 4,03 \\
\hline
\end{tabular}

Nota. Fonte: Resultados da Pesquisa.

Os dados indicam que os atributos mais bem avaliados quanto aos serviços atualmente prestados são o Atendimento educado e cortês, a consistência dos serviços em relação aos prazos, expresso em 
Percentual de entrega nos prazos, o Baixo nível de avarias nas cargas e a Ocorrência dos serviços conforme especificados. Porém a relação com a transportadora pode ser significativamente melhorada nos aspectos Disposição para ouvir e agir no caso de falhas e Informações sobre o status da carga, bem como na capilaridade do serviço (atendimento a qualquer Estado do Brasil).

A avaliação geral do serviço de transporte recebeu a menor média entre as empresas do cluster 1 e a maior nos clusters 3 e 4 . Algumas diferenças entre os clusters podem ser apontadas a partir dos dados reunidos na Tabela 9. A primeira delas fora mencionada, em que o cluster 1 avalia de maneira menos positiva quase todos os atributos, quando comparado com os demais clusters. No que diz respeito ao tempo para iniciar atendimento, as empresas do cluster 4 avaliam de maneira mais positiva. Já em relação ao Tempo para entregar o produto, essa avaliação mais positiva se desloca para o cluster 3. O atributo Disposição para ouvir e agir no caso de comunicação de falhas/problemas apresenta pior avaliação entre os atributos, sendo menos importante também para os clusters 3 e 4.

\section{Construtos dos serviços de transporte orientados pela escala de demanda de transporte}

Buscou-se identificar possíveis diferenças no que diz respeito à importância dos construtos do serviço de transporte, conforme o formato do serviço guiado pela escala do transporte - carga completa (Grupo 1) ou carga fracionada (Grupo 2). Neste caso, as notas médias indicam que as empresas que atendem ao varejo e aos consumidores finais, como é o caso do Grupo 2, tendem a atribuir maior importância a fatores ligados ao atendimento, demonstrando mais preocupação com a eventual ocorrência de problemas com o nível de serviço. Para as empresas do Grupo 1, por atenderem a grandes clientes, atributos do nível de serviço e integração à programação da cadeia, tais como o cumprimento de prazos, atendimento na hora marcada para descarregamento e integração em sistemas de produção enxuta, tornam o construto Segurança como o mais valorado, seguido da Relação com o cliente e Atendimento às necessidades especiais dos clientes (Tabela 11). Porém, o teste para a diferença das médias não demonstrou diferença estatística.

Tabela 11

\section{Importância dos Construtos do Serviço de Transporte para os Grupos*}

\begin{tabular}{lcc}
\hline Construtos & Grupo 1 & Grupo 2 \\
\hline Tempo & 4,51 & 4,32 \\
Segurança (no transporte e na carga) & 4,63 & 4,60 \\
Confiabilidade & 4,64 & 4,55 \\
Preço & 4,33 & 4,18 \\
Relação com o cliente & 4,29 & 4,34 \\
Atendimento às necessidades especiais dos clientes & 4,32 & 4,52 \\
\hline
\end{tabular}

Nota. *Diferenças das médias não-significante ao nível de 5\%.

Fonte: Dados da Pesquisa.

\section{Avaliação geral dos serviços de transporte pelos embarcadores}

Para se ter um panorama geral da importância atribuída a determinados atributos e à avaliação deles, foi elaborada uma matriz de resultado que indica o gap existente entre o que o embarcador valoriza (importância) e o que lhe é entregue (avaliação), como pode ser observado na Tabela 12. Nesta tabela, é possível verificar que os atributos relativos ao construto Confiabilidade têm maior importância para as empresas estudadas, seguidos de perto por aqueles relativos à Segurança.

A grandeza do hiato (RES) entre a importância do atributo e a avaliação do serviço prestado em relação àquele mesmo tributo indica pontos a serem melhorados no serviço de transporte. Observados 
os valores relativos aos atributos, é possível verificar forte hiato nos construtos Preço e Relação com o cliente. Dentro do aspecto Preço, a lacuna mais aguda diz respeito às políticas de descontos das transportadoras, seguido pelo preço do frete. Mesmo com pouca importância relativa, os embarcadores estão insatisfeitos com os preços dos serviços.

Tabela 12

Matriz de Resultado (RES): Importância (IMP) versus Avaliação (AVA)

\begin{tabular}{|c|c|c|c|c|c|c|c|}
\hline Construtos & Atributos & IMP & AVA & RES & IMP & AVA & RES \\
\hline \multirow{5}{*}{ Segurança } & Prevenção de roubo & 5,56 & 4,84 & $-0,72$ & \multirow{5}{*}{5,55} & \multirow{5}{*}{4,80} & \multirow{5}{*}{$-0,75$} \\
\hline & Prevenção de avarias & 5,58 & 4,64 & $-0,94$ & & & \\
\hline & Estado de conservação dos caminhões & 5,41 & 4,95 & $-0,46$ & & & \\
\hline & Prevenção de acidentes & 5,48 & 4,84 & $-0,64$ & & & \\
\hline & Ressarcimento de prejuízos & 5,73 & 4,72 & $-1,01$ & & & \\
\hline \multirow{5}{*}{ Confiabilidade } & Cumprimento do contrato & 5,61 & 5,12 & $-0,49$ & \multirow{5}{*}{5,67} & \multirow{5}{*}{5,05} & \multirow{5}{*}{$-0,62$} \\
\hline & Regularidade dos serviço & 5,58 & 5,10 & $-0,48$ & & & \\
\hline & Capacidade e disponib. Caminhões & 5,50 & 4,89 & $-0,61$ & & & \\
\hline & Pontualidade na entrega & 5,81 & 4,94 & $-0,87$ & & & \\
\hline & Integridade da carga & 5,84 & 5,22 & $-0,62$ & & & \\
\hline \multirow{4}{*}{ Preço } & Valores dos fretes & 5,56 & 4,36 & $-1,20$ & \multirow{4}{*}{5,40} & \multirow{4}{*}{4,32} & \multirow{4}{*}{$-1,07$} \\
\hline & Políticas de descontos & 5,33 & 3,85 & $-1,47$ & & & \\
\hline & Prazos de pagamento & 5,29 & 4,47 & $-0,82$ & & & \\
\hline & Rivalidade entre concorrentes & 5,42 & 4,62 & $-0,80$ & & & \\
\hline \multirow{6}{*}{$\begin{array}{l}\text { Relação do } \\
\text { Cliente }\end{array}$} & $\begin{array}{l}\text { Facilidade comunicação } \\
\text { c/ transportadora }\end{array}$ & 5,68 & 5,08 & $-0,60$ & \multirow{6}{*}{5,52} & \multirow{6}{*}{4,63} & \multirow{6}{*}{$-0,89$} \\
\hline & Agilidade do serviço & 5,70 & 4,97 & $-0,74$ & & & \\
\hline & Autonomia do responsável no atendimento & 5,54 & 4,63 & $-0,91$ & & & \\
\hline & Eficiência na solução de problemas & 5,67 & 4,53 & $-1,14$ & & & \\
\hline & Sistema de rastreamento da carga & 5,20 & 3,97 & $-1,23$ & & & \\
\hline & Cooperação técnica com clientes & 5,33 & 4,60 & $-0,73$ & & & \\
\hline
\end{tabular}

Nota. Fonte: Resultados da Pesquisa.

Quanto à Relação com cliente, as empresas estudadas indicam ser mais falha a eficiência na solução de problemas, acompanhada pela ineficiência dos sistemas de rastreamento da carga. Apesar das particularidades, vale destacar que em nenhum dos atributos ou dimensões as avaliações foram condizentes com os níveis de importância atribuídos pelos entrevistados, o que indica a necessidade de atuação das transportadoras a fim de reduzir este hiato.

Todavia os valores apresentados servem também para estimar uma avaliação geral do serviço prestado, considerando a importância dos elementos e suas avaliações. Neste caso, a satisfação das empresas em relação ao serviço prestado pelas transportadoras é $86 \%$, o que parece ser bastante relevante. 


\section{Considerações Finais}

Este artigo procurou entender como os embarcadores de pequeno e médio portes formam suas expectativas quanto aos serviços, como estas diferem de acordo suas necessidades sob o ponto de vista dos seus clientes a serem atendidos e como estes serviços são avaliados.

Os resultados indicaram que os construtos dos serviços de transporte mais valorizados pelas empresas embarcadoras foram, na ordem, Segurança, Confiabilidade, Tempo, Preço, Atendimento às necessidades especiais dos clientes e Relação com o cliente. Ou seja, os embarcadores manifestaram interesse direto na eficácia do serviço, apontando construtos de relacionamentos mais estreitos e até colaborativos como relativamente menos importantes.

Porém foram detectadas diferenças nas prioridades, conforme os agrupamentos orientados pelas características dos clientes. Empresas que têm como clientes típicos indústrias e de distribuidores, em todo o território, têm como fator mais relevante a Confiabilidade no serviço prestado pelas transportadoras. Por sua vez, empresas que atendem ao varejo local priorizam o Tempo da prestação dos serviços. Mas a Segurança é o construto mais valorizado, em linhas gerais, para as empresas que atendem ao varejo nacional, consumidor final, atacadistas e indústrias nacionais.

Por outro lado, o preço (frete) não foi uma variável que preponderou em qualquer cluster. Ao lado de Confiabilidade e Atenção às necessidades especiais, o Preço apenas é uma preocupação da mais alta relevância, à medida que os volumes dos pedidos aumentam. Ou seja, nas situações de maiores volumes de embarque, as empresas passam a dedicar atenção ao planejamento, desempenho, formatação do nível de serviço e negociação de preços, à medida que os volumes a serem movimentados aumentam.

Desta forma, os embarcadores esperam que o desempenho do transporte reforce o relacionamento com seus clientes. Em linhas gerais, o transporte parece ser estratégico, demandando atenção especial, apenas quando é o suporte para operações de altos valores financeiros. Desta forma, a negociação para compra de serviços de transporte tem características transacionais, sem interesse de aproximação e estreitamento de relacionamentos. A atenção dada à contratação do transporte equivale ao de uma transação que se encerra no próprio ato. Ou seja, é feita gestão de compras e não de transportes.

O relacionamento com a transportadora e a disposição dos embarcadores em implementar alianças torna-se importante apenas nos casos em que são atendidos grandes clientes. Nesta situação, parece ser condição para se manter na cadeia de negócios o cumprimento de prazos, atendimento com hora marcada para descarregamento e integração aos sistemas de produção enxuta, uma vez que é prática destes setores selecionar fornecedores com base em desempenho em vários critérios, entre os quais a logística.

Eles têm a necessidade de formar parcerias sólidas para também solidificar sua posição na cadeia. Nestes casos, as empresas ficam mais satisfeitas quanto aos serviços de transporte, indicando que relacionamentos mais colaborativos e estreitos implicam serviços de transporte planejados e integrados à estratégia de negócios das empresas. Em linhas gerais, a atenção dedicada ao transporte é de uma compra típica da empresa, e não como um componente da logística, uma fonte possível para definição de estratégia da empresa.

A importância atribuída aos construtos Segurança e Confiabilidade pode também ajudar a compreender a razão de muitas empresas manterem frota própria ou instituírem contratos para a movimentação de seus produtos. Para casos especiais, isto é, produtos com algumas especificidades, tais como perecíveis ou de muito alto valor agregado, de escala muito grande ou que atendam a cadeias de suprimentos, em que os indicadores da logística sejam muito relevantes na avaliação dos fornecedores, os embarcadores podem avaliar que o risco dos serviços prestados por operadores ocorram em níveis muito elevados, o que faz com que seja preferível ter o domínio destes serviços sob 
sua frota ou na forma de contratos rigorosos. Mas, como o nível de satisfação dos embarcadores é relativamente alto, estas são práticas que não predominam no mercado.

Embora a lacuna de conhecimento quanto às estratégias logísticas próprias ao universo de pequenas e médias tenha motivado tal pesquisa, reconhecem-se como limitações deste estudo as dificuldades de contextualizá-lo no universo empresarial mais amplo. Por isso, recomenda-se que estudos desta natureza também devem ser desenvolvidos para empresas de maior porte, para que haja o adequado contraponto analítico e se aprimore também o aprofundamento do tema na área acadêmica no Brasil. Ademais, a importância captada que se dá aos construtos reacende a necessidade de compreender as estratégias de transporte das empresas, principalmente no que diz respeito à razão de as empresas decidirem por manter frota própria ou instituírem contratos para suas operações.

\section{Artigo recebido em 09.04.2011. Aprovado em 13.09.2011.}

\section{Referências}

Agência Nacional de Transporte Terrestre. (2005). Pesquisa de avaliação da satisfação dos usuários dos serviços das empresas do transporte terrestre. Brasília: Autor.

Ballou, R. (2006). Revenue estimation for logistics customer service offerings. The International Journal of Logistics Management, 17(1), 21-37. doi: 10.1108/09574090610663419

Banco Internacional de Reconstrução e Desenvolvimento; Agência Nacional de Transporte Terrestre. (2006). Avaliação da demanda do transporte ferroviário de cargas no Brasil (Estudo relativo ao Empréstimo No BR-4.188/BIRD). Brasília: ANTT.

Bowersox, D. J., Closs, D. J., \& Cooper, M. B. (2002). Supply chain logistics management (Series Operations and Decision Sciences). New York: Mcgraw-Hill.

Cervo, L. A., Bervian, P. A., \& Silva, R. da (2007). Metodologia cientifica (6a ed.). São Paulo: Pearson Prentice Hall.

Collis, J., \& Hussey, R. (2009). Business research: a practical guide for undergraduate and postgraduate students (3a ed.). Basingstoke: Palgrave Macmillan.

Creswell, J. W. (2003). Research design: qualitative \& quantitative, and mixed methods approaches. (2a ed.). Thousand Oaks: Sage.

Dillon, W. R., \& Goldstein, M. (1984). Multivariate analysis. Methods and applications. New York: John Wiley \& Sons.

Gasse, Y. (1982). The modern entrepreneur: attributes and functions. Gestion, 7(4), 3-10.

Grant, D. B. (2005). The transaction - relationship dichotomy in logistics and supply chain management. Supply Chain Forum: an International Journal, 6(2), 38-48.

Hair, J. F., Anderson, R. E., Tatham, R. L., \& Black, W. C. (2005). Multivariate data analysis. New Jersey: Prentice Hall.

Holter, A. R., Grant, D. B., Ritchie, J., \& Shaw, N. (2008). A framework for purchasing transport services in small and medium size enterprises. International Journal of Physical Distribution \& Logistics Management, 38(1), 21-38. doi: 10.1108/09600030810857193

Malhotra, N. (2001). Marketing research: an applied orientation. New Jersey: Prentice Hall. 
Mason, S. J., Ribera, M. P., Farris, J. A., \& Kirk, R. G. (2003). Integrating the warehousing and transportation functions of the supply chain. Transportation Research Part E: Logistics and Transportation Review, 39(2), 141-159. doi: 10.1016/S1366-5545(02)00043-1

McCann, P. (2001). A proof of the relationship between optimal vehicle size, haulage length and the structure of distance-transport costs. Transportation Research Part A: Policy and Practice, 35(8), 671-693. doi: 10.1016/S0965-8564(00)00011-2

Meixell, M., \& Norbis, M. (2008). A review of the transportation mode choice and carrier selection literature. The International Journal of Logistics Management, 19(2), 183-211. doi: 10.1108/09574090810895951

Neuschel, R. P., \& Russell, D. M. (1998). Customer driven marketing in the transportation/logistics industry. International Journal of Logistics Management, 9(2), 99-106. doi: $10.1108 / 09574099810805799$

Ng, B., Ferrin, B. G., \& Pearson, J. N. (1997). The role of purchasing/transportation in cycle time reduction. International Journal of Operation and Production Management, 17(6), 574-591. doi: 10.1108/01443579710167267

Novaes, A. G. (2001). Logística e gerenciamento da cadeia de distribuição. Rio de Janeiro: Campus.

Pappu, M., Mundy, R. M., \& Paswan, A. (2001, April). New channel dynamics: an investigation of transport services. Anais do International Annual IPSERA Conference, Jönköping, Sweden, 10.

Schluter, M., \& Senna, L. A. S. (1999, novembro). As decisões de aquisição de serviços de transportes do pequeno varejista. Anais do Congresso de Ensino e Pesquisa em Transportes, São Carlos, SP, Brasil, 13.

Serviço de Apoio às Micro e Pequenas Empresas. (2009). Small and medium-sized enterprises support Brazilian agency. Boletim Estatístico das Micro e Pequenas Empresas. Recuperado em 13 outubro, 2010, de http:// http://www.sebraemg.com.br/arquivos/parasuaempresa/boletimestatisticompe/boletim_estatistic o_mpe.pdf

Skinner, W. (1969). Manufacturing - missing link in corporate strategy. Harvard Business Review, 47(3), 136-145.

Stone, H., \& Sidel, J. L. (1993). Sensory evaluation practices. London: Academic Press.

Valente, A. M., Passaglia, E., \& Novaes, A. G. (2008). Gerenciamento de transporte e frotas. São Paulo: Ed. Pioneira.

Yin, R. K. (1994). Case study research: design and methods. Thousands Oaks: Sage Publications. 